\title{
Ações intersetoriais realizadas pelos agentes comunitários de saúde em um município de pequeno porte do Espírito Santo
}

\author{
Intersectorial actions carried out by community health agents in the municipality of a small \\ municipality located in the state of Espírito Santo
}

Acciones intersectoriales realizadas por agentes comunitarios de salud en el municipio de un pequeño municipio ubicado en el estado de Espírito Santo

Zileidi Santos Faria Souza ${ }^{1 *}$, Maristela Dalbello-Araujo'.

\begin{abstract}
RESUMO
Objetivo: Identificar quais ações intersetoriais são desenvolvidas pelos Agentes Comunitários de Saúde (ACS's) de um município de pequeno porte localizado no estado do Espírito Santo, segundo as perspectivas dos mesmos. Métodos: Trata-se de um estudo descritivo exploratório de abordagem qualitativa, realizada com cinco ACS's atuantes no município há pelo menos um ano, sendo quatro do sexo feminino e um do sexo masculino. A coleta de dados foi feita através da realização de entrevistas individuais semiestruturadas, analisadas segundo a técnica de análise de conteúdo proposta por Bardin. Resultados: De forma predominante os ACS's entrevistados entendem por ações intersetoriais práticas que integram outros órgãos e serviços, numa perspectiva de trabalho em rede. Tais ações são desenvolvidas a partir das situações que são detectadas durante as visitas domiciliares, e estas por vezes não se concretizam em razão das burocracias. Dentre as ações intersetoriais desenvolvidas, recebeu destaque aquelas que tratam de indivíduos e famílias que apresentam algum tipo de vulnerabilidade social. Nem todos as políticas públicas do município são receptivas para o desenvolvimento destas ações. Conclusão: Os ACS's possuem uma clara compreensão do que venha ser uma prática intersetorial, e que por ser assim, tem atuado nesta perspectiva, por meio da interlocução com as demais políticas públicas do município.
\end{abstract}

Palavras-chave: Atenção primária à saúde, Estratégia saúde da família, Agente comunitário de saúde, Intersetorialidade.

\begin{abstract}
Objective: Identify which intersectoral actions are developed by Community Health Agents (ACS's) of a small municipality located in the state of Espírito Santo, according to their perspectives. Methods: This is an exploratory descriptive study with a qualitative approach, carried out with five ACSs working in the municipality for at least one year, four female and one male. Data collection was carried out through semi-structured individual interviews, analyzed according to the content analysis technique proposed by Bardin. Results: Predominantly, the CHAs interviewed understand practical intersectoral actions that integrate other bodies and services, in a networking perspective. Such actions are developed from situations that are detected during home visits, and these sometimes do not materialize due to bureaucracy. Among the intersectoral actions developed, those that deal with individuals and families who present some type of social vulnerability were highlighted. Not all public policies in the municipality are receptive to the development of these actions. Conclusion: The ACS's have a clear understanding of what an intersectoral practice will be, and that because it is so, it has acted in this perspective, through dialogue with the other public policies of the municipality.
\end{abstract}

Keywords: Primary health care, Family health strategy, Community health workers, Intersectoral collaboration.

1.Escola Superior de Ciências da Santa de Misericórdia (EMESCAM), Vitória - ES.
*E-mail: zileidifariasouza@hotmail.com 


\section{RESUMEN}

Objetivo: Identificar qué acciones intersectoriales son desarrolladas por los Agentes Comunitarios de Salud (ACS) de un pequeño municipio ubicado en el estado de Espírito Santo, según sus perspectivas. Métodos: Se trata de un estudio descriptivo exploratorio con abordaje cualitativo, realizado con cinco ACS que actúan en el municipio hace al menos un año, cuatro del sexo femenino y un masculino. La recolección de datos se realizó a través de entrevistas individuales semiestructuradas, analizadas según la técnica de análisis de contenido propuesta por Bardin. Resultados: Predominantemente, los ACS entrevistados comprenden acciones prácticas intersectoriales que integran otras instancias y servicios, en una perspectiva de trabajo en red. Dichas acciones se desarrollan a partir de situaciones que se detectan durante las visitas domiciliarias, y que en ocasiones no se concretan por la burocracia. Entre las acciones intersectoriales desarrolladas, se destacaron aquellas que atienden a personas y familias que presentan algún tipo de vulnerabilidad social. No todas las políticas públicas del municipio son receptivas al desarrollo de estas acciones. Conclusión: Las ACS tienen claro lo que será una práctica intersectorial, y que por serlo ha actuado en esa perspectiva, a través del diálogo con las demás políticas públicas del municipio.

Palabras clave: Atención primaria de salud, Estrategia de salud familiar, Agente comunitario de salud, Colaboración intersectorial.

\section{INTRODUÇÃO}

O Agente Comunitário de Saúde (ACS) é considerado como um trabalhador indispensável na Atenção Básica $(A B)$ já que representa um elo entre a comunidade e os serviços de saúde. Neste contexto, a $A B$ é entendida como um agrupamento de práticas de saúde, dirigidas aos indivíduos e as coletividades, objetivando a promoção e a proteção da saúde, a prevenção de agravos, o diagnóstico, e, por conseguinte, o tratamento e a recuperação, com vistas a manutenção da saúde do sujeito (MINISTÉRIO DA SAÚDE, 2011).

Domingues CC, et al. (2018) destacam a importância dos ACS na comunicação entre a equipe da AB e os usuários, na qual permeia o acesso da população adstrita aos serviços de saúde. Estes profissionais, se defrontam com problemáticas que transcendem o campo da saúde, na medida em que, para serem resolvidos, exigem atenção à diversos elementos das condições de vida da população, implicando assim, na adoção de uma ação intersetorial.

Junqueira LAP (2004) assinala que o conceito de intersetorialidade está acoplado a ideia de integração, de território, de equidade, enfim, dos direitos sociais. Trata-se de uma nova forma de tratar dos problemas sociais, que busca superar a fragmentação das políticas, concebendo o cidadão em sua totalidade.

A importância de pesquisar as ações intersetoriais realizadas pelos ACS se justifica por tratar de um tema que é de atribuição deste, conforme prevê a Portaria ํo 2.436, de 21 de setembro de 2017, porém, existem obstáculos que dificultam sua realização, dos quais precisam ser aprofundados (MINISTÉRIO DA SAÚDE, 2017).

Ante ao exposto, foi delimitado como objetivo geral identificar quais ações intersetoriais são desenvolvidas pelos ACS's de um município de pequeno porte localizado no estado do Espírito Santo, segundo as perspectivas dos mesmos. Já como objetivos específicos, investigar a concepção que os ACS's entrevistados possuem sobre ações intersetoriais; descrever os aspectos principais das atividades dos ACS's no município pesquisado; e, investigar as principais dificuldades que os ACS's se deparam ao realizarem ações intersetoriais.

\section{MÉTODOS}

Trata-se de uma pesquisa descritiva exploratória de abordagem qualitativa, no qual participaram cinco ACS's atuantes no município há pelo menos um ano, sendo quatro do sexo feminino e um do sexo masculino. Estes, por sua vez, foram designados pelas coordenações das Estratégias Saúde da Família (ESF) do município estudado, para serem o porta-voz dos demais ACS's de sua equipe, posto que, em razão da pandeia causada pela Covid-19 não foi possível a realização das entrevistas diretamente com todos os ACS's atuantes nas ESF do município em tela. 
Assim, os mesmos, receberam um roteiro com nove questões (APENDICE A) e se reuniram com os demais ACS's de sua Equipe para discutir as questões abordadas pelo pesquisador. Posteriormente, no período de agosto a setembro/2020, ocorreram as entrevistas com os cinco ACS's que responderam as nove questões com base nas informações coletadas com os demais ACS's de sua equipe. Destaca-se que, estas entrevistas foram gravadas, transcritas, e analisadas por meio da técnica de análise de conteúdo (BARDIN, 2011).

Impende realçar também que, visando assegurar o anonimato dos entrevistados, estes serão identificados nos recortes das entrevistas pela nomenclatura "equipe" seguida do número correspondente a qual equipe o ACS representou, já que, no total, o município conta com cinto equipes.

Insta frisar ainda que, a pesquisa empreendida recebeu aprovação do Comitê de Ética em Pesquisa (CEP) da Escola Superior de Ciências da Santa Casa de Misericórdia (EMESCAM), ficando registrada sob o número de parecer $n^{\circ} 3.730 .399$.

\title{
RESULTADOS E DISCUSSÃO
}

\section{A concepção que os ACS's entrevistados possuem sobre ações intersetoriais}

Após análise das entrevistas, foi possível verificar que de forma predominante os ACS's entrevistados entendem por ações intersetoriais práticas que integram outros órgãos e serviços, numa perspectiva de trabalho em rede.

\begin{abstract}
"Ao meu entender são as ações que desenvolvemos com dois ou mais órgãos, com o objetivo de melhoria da qualidade de vida das pessoas que necessitam dos serviços de determinado órgão. É a união de vários setores e esforço para alcançar recursos e melhorias para a população" (EQUIPE 2).

"É a integração de diversos setores, e ações em um atendimento na rede de serviços com recursos disponíveis para ajudar a resolver os problemas. Ações intersetoriais é trabalhar em conjunto com outros setores como, esporte, lazer, cultura, educação, ações em saúde, transformar o local de trabalho num ambiente saudável, é uma união de esforços para alcançar as melhorias e ter um resultado para melhorias das condições de vida da comunidade" (EQUIPE 4).
\end{abstract}

Assim, em consonância com a pesquisa de Dias MAS, et al. (2014), nota-se que os entrevistados concebem as ações intersetoriais como aquelas que são articuladas e integradas e que resultam em benefícios para as condições de vida dos usuários e da comunidade como um todo. A pesquisa de Souza MCO (2020) também detectou que os participantes de seu estudo associaram a prática intersetorial a integração dos setores e ao trabalho em rede.

Sobre o trabalho numa perspectiva de rede, Pedruzzi M e Agreli HF (2018) citam que as redes se constituem enquanto um modo de enfrentamento dos sistemas fragmentados de atenção à saúde, que amplia a possibilidade de ação intersetorial para além do contexto da equipe de saúde, mas também para outras equipes que compõem a rede, em parceria com usuários e a comunidade adstrita.

Para os sujeitos partícipes desta investigação, tais ações são desenvolvidas a partir das situações que são detectadas durante as visitas domiciliares, na qual, diante destas, são buscadas parcerias capazes de solucionar os problemas que são identificados, tal como prevê a Portaria ํㅜ 2.436, de 21 de setembro de 2017 (MINISTÉRIO DA SAÚDE, 2017).

"Através das visitas domiciliares, buscando parcerias favoráveis que resolvam os problemas identificados na comunidade" (EQUIPE 1).

"Identifico através das visitas, conhecendo a necessidade de cada um ou da comunidade" (EQUIPE 4).

Este achado também foi encontrado na pesquisa de Fracolli LA, et al. (2015) que mencionaram que durante as visitas domiciliares realizadas pelos ACS's estes se deparam com questões que extrapola o setor de saúde, sendo, por vezes, questões complexas que impõem a necessidade de realização de ações intersetoriais e, uma visão ampliada do conceito de saúde, incluindo os determinantes e condicionantes sociais envolvidos neste conceito. 
Ressalta-se que, embora as ações intersetoriais sejam propostas pelos ACS's do município estudado, estas, muitas vezes, não se concretizam em razão das burocracias, conforme pode-se perceber a partir do relato abaixo e, este empecilho também foi encontrado na pesquisa de Souza MCO (2020) no que tange a efetivação das ações intersetoriais:

"As dificuldades que impedem de realizar ações intersetoriais são as burocracias através de hierarquias que existem em determinados setores onde fica um jogo de empurra. Onde muitas vezes não conseguimos falar com a pessoa responsável, como o secretário, por exemplo, e na maioria das vezes não conseguimos resolver certos problemas que identificamos na nossa microárea" (EQUIPE 5).

Assim, cabe pontuar o pensamento de Santos NN (2011) que diz que dentre os objetivos da intersetorialidade encontra-se a horizontalização das relações entre os serviços com base na interdependência dos setores, de forma a romper com as variações da hierarquia burocrática setorial.

Dias MAS, et al. (2014) identificaram que, no contexto das ações intersetoriais desenvolvidas por profissionais ligados a ESF, a excessiva burocracia atrapalha o processo de efetivação de tais ações. No que se refere a isso, Inojosa RM (1998) analisa que a forma como o sistema governamental se encontra estruturado, o qual possui diversos escalões hierárquicos, reflete diretamente na qualidade dos serviços e produtos que o Estado dispõe para a Sociedade, influenciando também na efetivação da intersetorialidade.

Fracolli LA, et al. (2015) encontraram dado similar ao identificado nesta pesquisa, quando verificaram que muitos ACS's não conseguiam resolver os problemas identificados na comunidade por meio das ações intersetoriais, mesmo estas tendo sido propostas, destacando como motivo, a falta de articulação entre os setores, por exemplo. Estes autores acrescentam que a implantação da intersetorialidade exige uma decisão que é política, uma vez que implica em alterações na organização das estruturas de poder, requerendo assim, uma mudança na forma como está estruturado o trabalho, na lógica de governo existente, para que assim, os problemas existentes na comunidade possam ser prevenidos ou solucionados.

Cabe citar o que diz Ornelas AL e Teixeira MGC (2015), que ressaltam que para que a intersetorialidade aconteça de fato, é preciso que esta prática venha se sobrepor as hierarquias, encontrando eco suficiente nos outros setores, além do da saúde, para que assim possa se consolidar.

Já Carmo ME e Guizardi FL (2017), por sua vez, analisam que, as burocracias existentes no campo das Políticas Públicas, dificultam a atuação numa perspectiva intersetorial, e que pode se revelar como forma de resistência e, por vezes, apontar para mudanças substanciais no modelo do contexto do modelo organizacional, no qual, este é o motivo pelo qual o objetivo final de uma Política Pública, comum a todos os setores envolvidos, revela-se como o caminho mais apropriado para uma atuação intersetorial com menores empecilhos.

\section{Ações intersetoriais desenvolvidas pelos ACS's pesquisados}

Dentre as ações intersetoriais desenvolvidas, recebeu destaque aquelas que tratam de indivíduos e famílias que apresentam algum tipo de vulnerabilidade social.

"Quando nós nos deparamos com famílias que estão em vulnerabilidade social e que se enquadram no perfil do CRAS e demais necessidade que o usuário estiver, encaminhamos para esse setor" (EQUIPE 3).

"Identificamos a necessidade de desenvolver ações intersetoriais através das visitas domiciliares, onde encontramos famílias em situação de vulnerabilidade social e crianças e idosos com maus tratos, onde encaminhamos o caso aos setores responsáveis para estar atendendo esses usuários" (EQUIPE 5).

A atuação do ACS's na política de Assistência social pode ser atestada na pesquisa de Souza MCO (2020), que reforçou a importância da participação desta categoria profissional para a referida política, devido ao fato deste fazer parte do cotidiano das famílias que são acompanhadas por esta. A referida autora destacou que, embora haja a comunicação entre a Política Pública de Saúde e a de Assistência social, nota-se que é preciso que esta venha ser amadurecida, para que haja uma constante articulação intersetorial entre ambas. 
Domingues CC, et al. (2018) também destacam que a prática do ACS transcende o setor da saúde, uma vez que este sempre se depara com situações que exigem uma ação intersetorial, uma vez que estes reconhecem seu território de abrangência que, por sua vez, possibilita realizar o diagnóstico de grupos socialmente vulneráveis. Por isso, sua atuação em rede de atenção implica na articulação de diversos setores visando produzir saúde por intermédio do rompimento das dificuldades que se fazem presentes neste campo, no qual este rompimento propicia maior resolutividade e produção de um cuidado-cuidador. Além disso, as idas e vindas do usuário em busca de atendimento são diminuídas com o rompimento dessas dificuldades. Não obstante, muitas das necessidades da comunidade extrapolam a área estrita da saúde.

Riquinho DL, et al. (2018) citam que os ACS's são confrontados em sua prática com situações extremamente complexas, como por exemplo, os casos de diversos tipos de violências, como agressões, abuso sexual e abandono. Tais situações demostraram a delicada tarefa que é designada a estes profissionais que, por serem concebidos como representantes do Estado, precisam atuar também numa perspectiva de defesa dos indivíduos que buscam por segurança, como por exemplo, os casos de crianças ou idosos vitimados.

No que diz respeito às falas dos participantes acima, é valido destacar o que diz Maciel FBM, et al. (2020), que discorrem que as visitas domiciliares realizadas pelos ACS's são um valioso instrumento para que estes profissionais venham fazer um diagnóstico situacional das vulnerabilidades apresentadas pela família, permitindo identificar situações de extrema pobreza, insegurança alimentar, bem como, a existência de grupos vulneráveis. Deste modo, após identificar as famílias mais vulneráveis, o ACS pode estabelecer contato com outros serviços, como por exemplo, o serviço de proteção e o de assistência social, para que a família seja assistida nas demandas que foram identificadas.

Assim, embora o trabalho do ACS possa ser considerado como o do campo da saúde, este, por sua vez, engloba ações intersetoriais, como por exemplo, a articulação com o Centro de Referência de Assistência Social (CRAS) e com o Centro de Referência Especializado de Assistência Social (CREAS), a fim de buscar melhores estratégias que garantam a prevenção e a promoção da saúde das famílias de sua microárea (MACIEL FBM, et al., 2020).

Portanto, pode-se dizer que o ACS se encontra inserido na linha de frente da identificação de situações de riscos de violência, fazendo-se necessário um treinamento adequado para que estes possam desenvolver ações intersetoriais de prevenção e controle das violências que se fazem presentes em seu território (VIEIRAMEYER APG, et al., 2021).

As entrevistadas também fizeram menção a realização de palestras, a participação nos grupos do Programa Nacional de Hipertensão e Diabetes mellitus (HIPERDIA), a coleta de dados para o Sistema de Vigilância Alimentar e Nutricional (SISVAN) e a liberação da Ficha de Cadastro do Usuário (Ficha A) para o usuário, como sendo ações intersetoriais, quando na verdade, se referem a ações que são inerentes ao seu fazer diário. Deste modo, nota-se que os ACS não possuem expressiva clareza acerca das atribuições que Ihe são designadas, e que por isso intitulam como ações intersetoriais até mesmo aquelas que são de sua competência.

"Sempre desenvolvemos atividades nas escolas sobre saúde bucal e como ter bons hábitos alimentares, através de palestras com profissionais da equipe como: dentistas, enfermeiros e nutricionistas" (EQUIPE 2).

"Realizamos várias ações como: grupo de HIPERDIA, SISVAN nas escolas, pesagens e palestras com idosos entre outros, onde trabalhamos com o objetivo de analisar a dinâmica e com métodos de pesquisa e com novas ideias para obter resultados e concluir um bom trabalho onde colocamos em prática dentro e fora do setor saúde para termos superação dos problemas" (EQUIPE 4).

"Temos exemplo de ações intersetoriais como a liberação de ficha A (Ficha de cadastro do usuário), onde liberamos para o usuário quando solicitam por meio de protocolo para conseguir algum benefício, a pesagem da Bolsa Família e palestras nas escolas" (EQUIPE 5). 
Em se tratando das palestras realizadas pelos ACS's, juntamente com a equipe da ESF, Alonso CMC, et al. (2018) reforçam que tais ações são de grande relevância, tendo como efeito positivo a integração dos ACS's com os demais profissionais, possibilitando assim, o estabelecimento de atuações coletivas para o enfrentamento de determinados problemas identificados na comunidade.

Acerca disso, Dantas DSG, et al. (2018) refletem que o ACS é um ator de destaque para o campo da educação em saúde, uma vez que, a partir do desenvolvimento de práticas educativas, é possível haver transformações no campo da atenção à saúde individual e coletiva e que, mesmo sendo assim, nota-se que ainda não há grande investimento na qualificação deste, conforme também encontrado nos relatos dos ACS's entrevistados nesta pesquisa.

Soares AM, et al. (2020), corroborando com os resultados da presente pesquisa, verificaram que os ACS's pesquisados, além de realizar as visitas domiciliares, realizavam também palestras e eram atuantes nos grupos de Hiperdia.

Contudo, analisando a participação do ACS no Hiperdia, é preciso destacar o que diz a pesquisa de Souza MCO (2020), que também identificou que os profissionais entrevistados reconheciam ações realizadas por um determinado profissional em conjunto com demais profissionais da equipe de saúde como sendo ações intersetoriais, mas que na verdade se tratava de ações intrasetoriais e que expressavam a interdisciplinaridade, não podendo, portanto, ser confundidas como práticas intersetoriais.

O Hiperdia se refere a um sistema que visa o cadastramento e acompanhamento de hipertensos e diabéticos, onde os profissionais de saúde recebem a incumbência de ofertar aos usuários o atendimento e realizar o preenchimento dos dados neste sistema. Além disso, cabe a estes profissionais, programar e implementar ações de investigação e acompanhamento dos usuários, na qual, a educação em saúde precisa ser englobada em seu fazer cotidiano, exemplo dessa prática é a realização de palestras a fim de contribuir para que o usuário possa aderir ao tratamento, uma vez que este é visto como protagonista do seu processo de reabilitação (FILHA FSSC, et al., 2014).

Abordando sobre a atuação intersetorial do ACS no Programa Bolsa Família (PBF), essa prática também foi reconhecida pelos participantes da pesquisa conduzida por Souza MCO (2020) como sendo uma ação de caráter intersetorial que envolve a política de assistência social e a política de saúde.

Sobre o PBF, Moraes VD e Machado CV (2017) citam que existe uma complexidade no que se refere a gestão das condicionalidades do referido programa, uma vez que o processo engloba diferentes setores, como o da saúde, educação e assistência, além de envolver, na sua operacionalização, diversos atores.

Na concepção dos entrevistados, os setores que demonstram mais receptividade para o desenvolvimento das ações intersetoriais por eles desenvolvidas são: o CRAS; o CREAS; o Conselho Tutelar; a Secretaria Municipal de Habitação; a Secretaria Municipal de Educação e, a Secretaria Municipal de Transporte.

"Na verdade, deveriam ser todos setores, mas a nossa realidade não é bem essa, raro podemos contar com outros setores e servimos para fazer o trabalho deles como: bolsa família, cesta básica, saúde mental, infelizmente isso não acontece, hoje não podemos dizer que temos essa interação. Os setores que mais integram é a secretaria de assistência social (CRAS E CREAS), habitação e educação" (EQUIPE 4).

"Os setores que mais cooperam para a realização de ações intersetoriais é a educação, assistência social, conselho tutelar e transporte" (EQUIPE 5).

Deste modo, pode-se perceber, no discurso da entrevistada que representou a equipe 4 que, embora se reconheça a necessidade de integração da saúde com as demais secretarias do município, na prática essa integração não ocorre.

Assim, é preciso destacar o que diz Dias MAS, et al. (2014) que pontuam que, por vezes, verificam-se práticas que envolvem vários setores e que são rotuladas como se fossem intersetoriais, mas que, não estão interligadas de forma efetiva. 
Acerca disso, Anastácio E, et al. (2016) pontuam que, para que a intersetorialidade se efetive, é primordial que as diversas políticas públicas não trabalhem de forma isoladas, mas sim interligadas e interdependentes, para que ocorra interações capazes de proporcionar ganhos para todos os sujeitos envolvidos.

Analisando ainda a primeira fala descrita acima, nota-se que os entrevistados entendem que algumas atividades que realizam não são de sua competência, sendo esta percepção também identificada entre os ACS's entrevistados nas pesquisas de Riquinho DL, et al. (2018), Alonso CMC, et al. (2018) e Santos AC, et al. (2019).

A esse respeito, Riquinho DL, et al. (2018) refletem que o ACs's está inserido em um campo de indeterminações e incertezas, onde não se tem uma clara definição acerca de suas competências, saberes e habilidades. Essa indefinição contribui para que estes assumam atividades que se mostram distantes de seu lócus de intervenção, ou seja, o território vivido.

Em relação ao exposto, Alonso $\mathrm{CMC}$, et al. (2018) também dizem que não existe uma definição clara sobre as atribuições inerentes aos ACS's, resultando no excesso de funções desempenhadas por estes, das quais muitas delas não são de sua competência e outras são inseridas de forma gradual e oficial no rol de suas atribuições, como por exemplo: a pesagem das famílias para recadastramento do PBF.

Já em relação aos setores que demostram menor receptividade para o desenvolvimento das ações intersetoriais, as participantes deste estudo destacaram como sendo a Secretaria Municipal de Finanças; os setores privados; a Vigilância Epidemiológica; a Secretaria Municipal de Segurança Pública e, a Secretaria Municipal de Obras.

"Bom, para mim é o setor finanças, pois muitas vezes eles ouvem a gente, mas não colocam em prática nossas reivindicações" (EQUIPE 1).

"Os setores privados" (EQUIPE 2).

"Os setores mais difíceis de conversar é a vigilância epidemiológica, secretaria de segurança e secretaria de obras é a que temos menos acesso e as mais complicadas" (EQUIPE 5).

Em se tratando de intersetorialidade, é preciso dizer que a comunicação entre os setores envolvidos é de grande relevância e que a falta deste elemento impede que esta prática se efetive. Conforme expõe Warschauer M e CarvalhoYM (2014) a comunicação é a costura da ação intersetorial, ao realizar a articulação entre as partes, expondo os conflitos e podendo abrir caminhos para soluções. Cabe realçar que não apenas os setores públicos precisam estar envolvidos nas ações intersetoriais, mas também os setores privados, igrejas e associações comunitárias, conforme cita Dias MAS, et al. (2014).

Diante dos dados acima, é preciso que a gestão municipal implemente ações que possam fortalecer a integração entre os setores que são menos receptivos às ações intersetoriais propostas pelos ACS's do município estudado, a fim de que os munícipes sejam atendidos das demandas que são apresentadas a estes profissionais durante as visitas domiciliares.

\section{CONCLUSÃO}

Por meio desta, foi possível perceber que os ACS's pesquisados possuem uma clara compreensão do que venha ser uma prática intersetorial, e que por ser assim, tem atuado nesta perspectiva, por meio da interlocução com as demais políticas públicas do município. Entretanto, tal atuação é permeada por alguns percalços, por exemplo, a excessiva burocracia de determinados setores e pouca receptividade de alguns órgãos para que as ações propostas por estes profissionais sejam efetivadas. Destarte, esta pesquisa trouxe elementos que poderão servir de subsídios para a proposição, implementação e avaliação das políticas públicas do município estudado.

\section{REFERÊNCIAS}

1. ALONSO CMC, et al. Trabalho dos agentes comunitários de saúde na Estratégia Saúde da Família: metassíntese. Rev. Saúde Pública, 2018; 52: 1-13. 
2. ANASTÁCIO E. et al. Intersetorialidade entre as políticas públicas de assistência social e saúde: desafios para a promoção da saúde. Rev. Med. Minas Gerais, 2016; 26: 362-366.

3. BARDIN L. Análise de conteúdo. São Paulo: Edições 70, 2011; 229p.

4. CARMO ME, GUIZARDI FL. Desafios da intersetorialidade nas políticas públicas de saúde e assistência social: uma revisão do estado da arte. Physis: Revista de Saúde Coletiva, 2017; 27: 1265-1286.

5. DANTAS DSG, et al. A Formação dos agentes comunitários de saúde em educação popular: implicação na produção do cuidado na Estratégia Saúde da Família. Motricidade, 2018; 14: 157-163.

6. DIAS MAS, et al. Intersetorialidade e Estratégia Saúde da Família: tudo ou quase nada a ver? Ciência e Saúde coletiva, 2014; 19: 4371-4382.

7. DOMINGUES CC, et al. Potencialidades do processo de trabalho do Agente Comunitário de Saúde. Rev. Bras. Pesq. Saúde, 2018; 20: 145-154.

8. FILHA FSS, et al. Avaliação do controle de hipertensão e diabetes na Atenção Básica: perspectiva de profissionais e usuários. Saúde debate, 2014; 38: 265-278.

9. FRACOLLI LA, et al. Perspectivas das ações intersetoriais, de inclusão e participação sociais desenvolvidas por agentes comunitários de saúde. Rev. Bras Promoção saúde, 2015; 28: 82-85.

10. INOJOSA RM. Intersetorialidade e a configuração de um novo paradigma organizacional. RAP, 1998; 32 : 35-48.

11. JUNQUEIRA LAP. A gestão intersetorial das políticas sociais e o terceiro setor. Saúde e Sociedade, 2004; $13: 25-36$.

12. MACIEL FBM, et al. Agente comunitário de saúde: reflexões sobre o processo de trabalho em saúde em tempos de pandemia de Covid-19. Ciência \& Saúde Coletiva, 2020; 25: 4185-4195.

13. MINISTÉRIO DA SAÚDE. Política Nacional de Atenção Básica. Brasília: Ministério da Saúde, Secretaria de Atenção à Saúde, Departamento de Atenção Básica. 2011. Disponível em: http://189.28.128.100/dab/docs/publicacoes/geral/pnab.pdf. Acessado em: 05 de janeiro de 2021.

14. MINISTÉRIO DA SAÚDE. Portaria 2436, de 21 de setembro de 2017. Aprova a Política Nacional de Atenção Básica, estabelecendo a revisão de diretrizes para a organização da Atenção Básica, no âmbito do Sistema Único de Saúde (SUS). 2017. Disponível em: https://bvsms.saude.gov.br/bvs/saudelegis/gm/2017/prt2436_22_09_2017.html. Acessado em: 20 de janeiro de 2021.

15. MORAES VD, MACHADO CV. O Programa Bolsa Família e as condicionalidades de saúde: desafios da coordenação intergovernamental e intersetorial. Saúde debate, 2017; 41: 129-143.

16. ORNELAS AL, TEIXEIRA, MGC. Intersetorialidade ou diálogos setoriais? Reflexões a partir da experiência do Projeto Teias-Escola Manguinhos, Rio de Janeiro. Saúde debate, 2015; 39: 659-670.

17. PEDUZZI M, AGRELI HF. Trabalho em equipe e prática colaborativa na Atenção Primária à Saúde. Interface (Botucatu), 2018; 22: 1525-1534.

18. RIQUINHO DL, et al. O cotidiano de trabalho do agente comunitário de saúde: entre a dificuldade e a potência. Trab. Educ. Saúde, 2018; 16: 163-182.

19. SANTOS AC, et al. Agente Comunitário de Saúde: implicações dos custos humanos laborais na saúde do trabalhador. Physis: Revista de Saúde Coletiva, 2018; 28: 1-18.

20. SANTOS NN. A intersetorialidade como modelo de gestão das políticas de combate à pobreza no Brasil - O caso do Programa Bolsa Família no Município de Guarulhos. Dissertação (Mestrado em Administração Pública e Governo). Escola de Administração de Empresas de São Paulo da Fundação Getúlio Vargas, São Paulo, 2011; 166p.

21. SOARES AM, et al. Cuidado em saúde às populações rurais: perspectivas e práticas de agentes comunitários de saúde. Physis: Revista de Saúde Coletiva, 2020; 30: 300-332.

22. SOUZA MCO. Intersetorialidade entre as políticas de saúde e assistência social: possibilidades e limites em Presidente Kennedy- ES. Dissertação (Mestrado em Políticas Públicas e Desenvolvimento Local) . Escola Superior de Ciências da Santa Casa de Misericórdia, Vitória- ES, 2021; $121 \mathrm{p}$.

23. VIEIRA-MEYER APG. Violência e vulnerabilidade no território do agente comunitário de saúde: implicações no enfrentamento da COVID-19. Ciênc. Saúde coletiva, 2021; 26: 657-668.

24. WARSCHAUER M, CARVALHO YM. O conceito "Intersetorialidade": contribuições ao debate a partir do Programa Lazer e Saúde da Prefeitura de Santo André/SP. Saúde Soc., 2014; 23: 191-203. 